\title{
Deformation of coronary stent: coronary CT angiography
}

Keywords: Coronary artery disease - Percutaneous coronary intervention - Cardiac CT angiography - Optical coherence tomography

A 57-year-old male was admitted to hospital for study of exertional chest pain. The coronary CT angiography showed severe stenosis in both ramus intermedius (RI) and first obtuse marginal artery (OM1). The coronary angiography performed confirmed previous findings and showed moderate lesions in the left anterior descending artery (LAD) and right coronary artery as well. The patient underwent percutaneus coronary intervention with drug-eluting stent implantation in the proximal RI and balloon angioplasty of the OM1 after multiple unsuccessful attempts to advance the stent across proximal circumflex artery. Two days later the patient was readmitted for NSTEMI. The coronary angiogragphy did not find any new lesion, but the optical coherence tomography (OCT) exam showed a proximal deformation of the stent implanted in the proximal RI, that involved the left main coronary artery and was displaced toward the ostium of the circumflex artery, that was partially obstructed but mantained TIMI 3 flow (Figure 1). This was confirmed by a new coronary CT angiography (Figures 2 and 3). The stent deformation was thought to be caused by the failed attempts to push the stent across the ostium of the circumflex artery in the first procedure. The patient remained symptomatic with recurrent resting angina and dynamic EKG changes during hospitalization. The case was discussed by the Heart Team, and due to the anatomical complexity the patient finally underwent onpump coronary artery bypass grafting with left internal mammary artery bypass to LAD and sequential saphenous vein bypass to OM1 and RI.

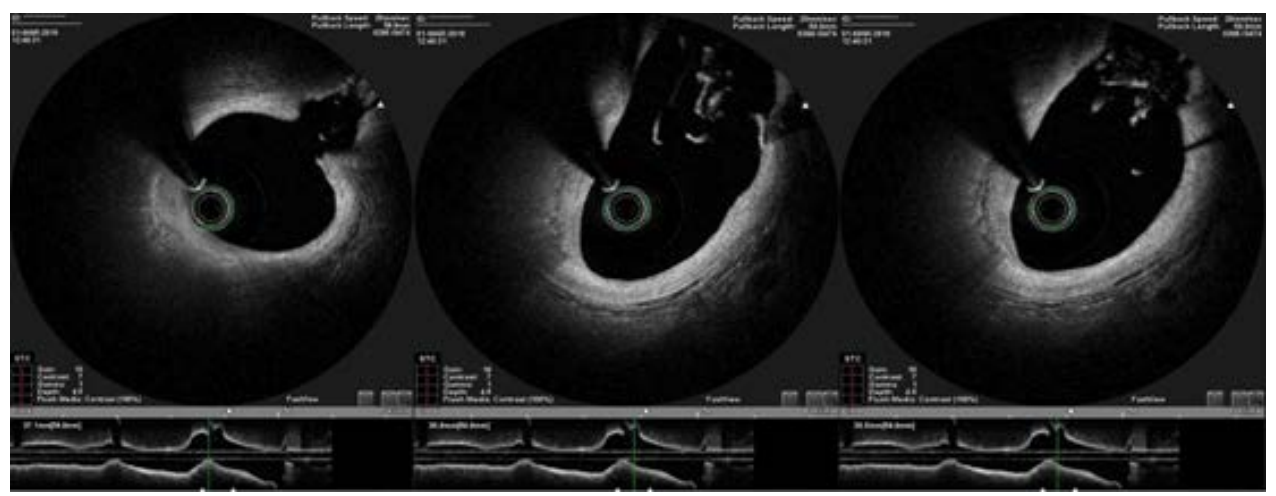

Figure 1: Coronary angiography 


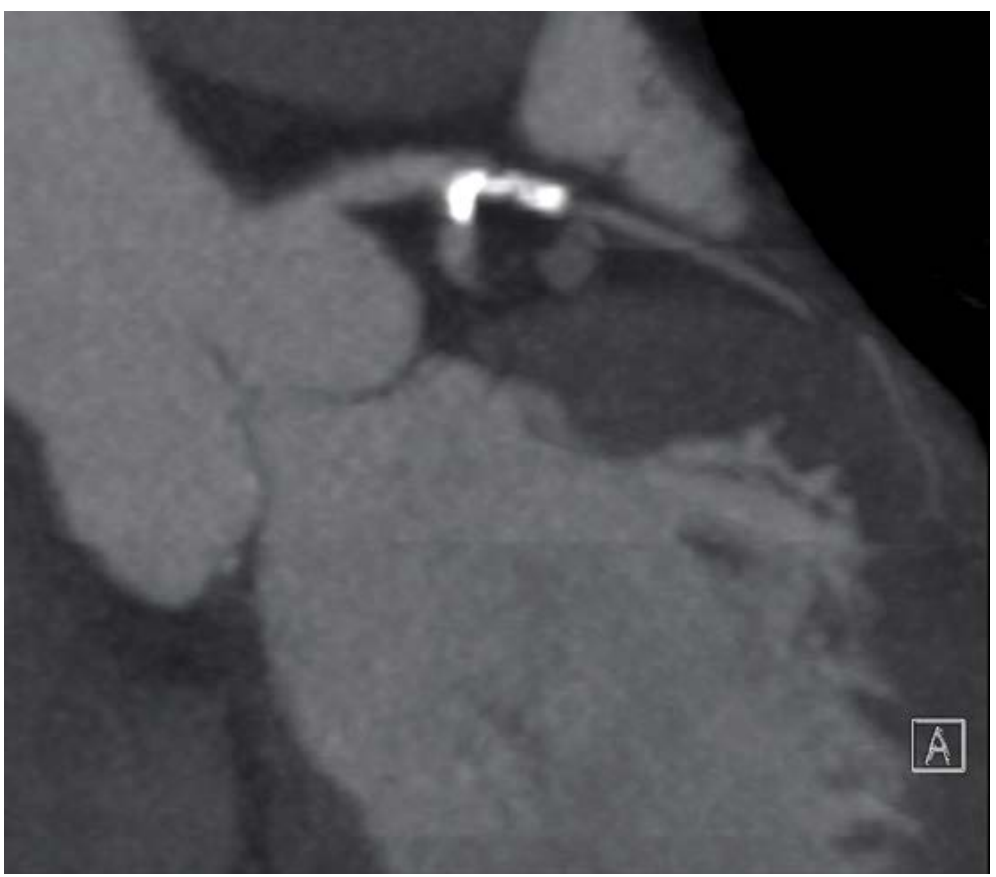

Figure 2: Coronary CT angiography

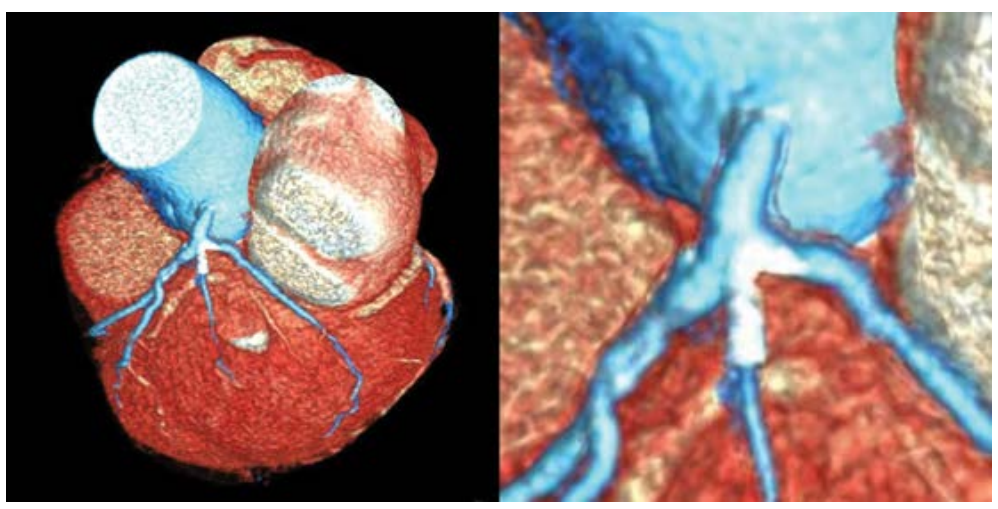

Figure 3: Coronary CT angiography 